\title{
PATRÓN POBLACIONAL PREHISPÁNICO EN MEDIAS AGUAS, VERACRUZ
}

\section{PREHISPANIC POPULATION PATTERN IN MEDIAS AGUAS, VERACRUZ}

\section{Roberto Lunagómez Reyes ${ }^{1}$}

tiozorro007@hotmail.com

\section{RESUMEN}

Medias Aguas se localiza dentro del Istmo de Tehuantepec, entre el océano Pacífico y el Golfo de México, este se inserta en el municipio de Sayula de Alemán, Veracruz, también se localiza entre dos sitios arqueológicos importantes: San Lorenzo y Las Limas, una parte fundamental de esta investigación tuvo como objetivo principal la interpretación de los mecanismos que intervinieron en el surgimiento, evolución y/o desintegración de la complejidad sociopolítica que caracterizaron a las ocupaciones humanas en la región, basándose metodológicamente en el estudio del Patrón de asentamiento regional empleando las técnicas de campo diseñadas para un reconocimiento sistemático e intensivo de superficie, el cual integra un área de estudio de $200 \mathrm{~km}^{2}$ en donde se registraron 124 sitios arqueológicos.

Palabras clave: Esteros, patrón de asentamiento, Mascarón de Medias Aguas.

\footnotetext{
${ }^{1}$ Museo de Antropología de Xalapa, Universidad Veracruzana, México.
} 


\begin{abstract}
Medias Aguas is located within the Istmo of Tehuantepec, between the Pacific Ocean and the Gulf of Mexico, it is inserted in the township from Sayula of Alemán, Veracruz, it is also located between two important archaeological sites: San Lorenzo and The Limas, a part the main objective of this research was to interpret the mechanisms that intervened in the emergence, evolution and / or disintegration of the sociopolitical complexity that characterized human occupations in the region, based methodologically on the study of the regional settlement pattern using the Field techniques designed for a systematic and intensive surface survey, which integrates a $200 \mathrm{~km}^{2}$ study area where 124 archaeological sites were recorded.
\end{abstract}

Keywords: Estuaries, settlement pattern, Mask of half waters.

\title{
CONTEXTO DE LA INVESTIGACIÓN²
}

La región de Medias Aguas, foco del presente estudio, se ubica dentro del Istmo de Tehuantepec, el cual destaca como la zona más estrecha del territorio mexicano localizada entre el océano Pacífico y el Golfo de México; esta estrecha faja continental en su porción norte es conocida como el Istmo Veracruzano (Münch 1983: 15), el cual se caracteriza por dos ríos principales, el Coatzacoalcos y el San Juan; en esta porción de la República Mexicana se encuentran ocasionales lomeríos de tierras elevadas y amplias planicies aluviales inundadas estacionalmente por un sinnúmero de ríos, esteros ${ }^{3}$ y arroyos tributarios de los

\footnotetext{
${ }^{2}$ Esta investigación fue posible gracias al apoyo económico, logístico de CONACYT, Ann Cyphers, FAMSI, Pierre Masson, Hiroshi Minami, NGS y a todos los miembros del Proyecto Arqueológico Medias Aguas, entre ellos: Virginia Arieta, Yiro Cisneros, Hirokazu Kotegawa, Mitsuru Kurosaki, Xóchitl León, Nelly Núñez y Ericka Ortíz.

${ }^{3}$ Cabe aclarar que el término "estero" se utiliza en la región y a lo largo del presente texto para hacer referencia a un río de corriente lenta de tipo pantanoso.
} 
grandes ríos mencionados. En la parte central del Istmo Veracruzano se localiza la región de Medias Aguas, la cual se inserta en el municipio de Sayula de Alemán, Veracruz; también se ubica entre dos sitios arqueológicos importantes, San Lorenzo localizado $20 \mathrm{~km}$ aproximadamente al noreste y Las Limas ubicado cerca de $40 \mathrm{~km}$ al sureste (Figura 1).

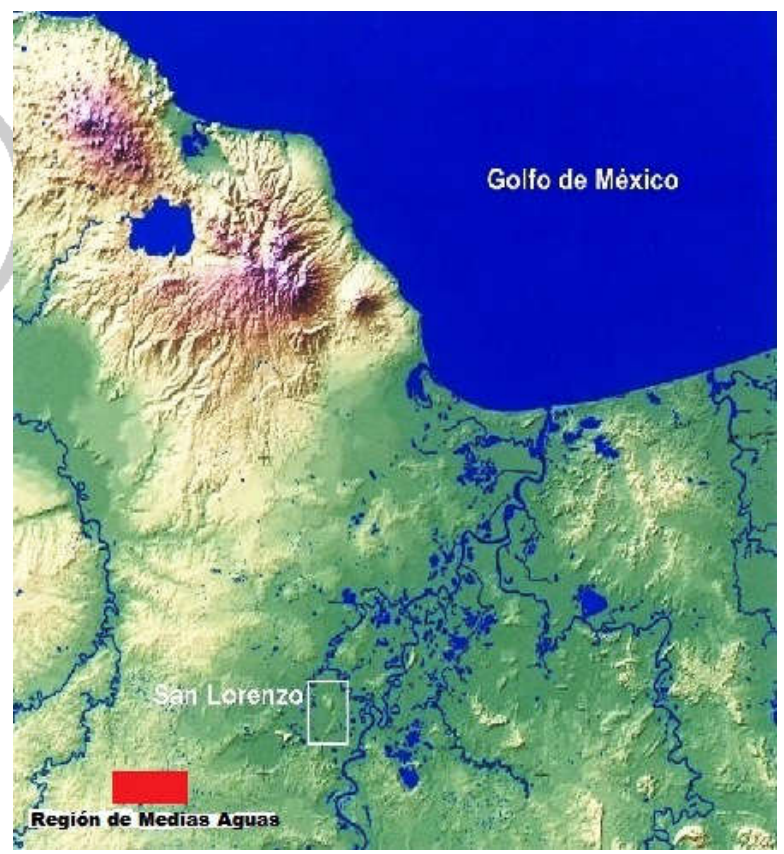

Figura 1: Ubicación geográfica de Medias Aguas, Veracruz (modificada de Cyphers 2012).

A grandes rasgos la región está compuesta por: a) una banda de tierras elevadas con dirección este-oeste, sólo interrumpida por un cerro alto que sobresale dentro del paisaje regional y 2) zonas bajas inundables de carácter pantanoso cruzadas por los esteros El Juile y Monte Obscuro, así como sus tributarios. Hay que notar 
que en la región no aparecen ríos de mediano calado como en la cuenca media del río Coatzacoalcos hacia el oriente o de ríos encajonados como La Trinidad, tributario del río San Juan hacia el occidente.

La presente investigación tuvo como objetivo principal la interpretación de los mecanismos que intervinieron en el surgimiento, evolución y/o desintegración de la complejidad sociopolítica que caracterizaron a las ocupaciones humanas en la región de estudio a nivel diacrónico. Se basó metodológicamente en el estudio del Patrón de asentamiento regional, empleando las técnicas de campo diseñadas para un reconocimiento sistemático e intensivo de superficie, integra un área de estudio de $200 \mathrm{~km}^{2}$ en donde se registraron 124 sitios arqueológicos, y complementa la exploración regional al añadirse a otros estudios en el sur de Veracruz como los de Kruger (1996); Borstein (2001); Symonds et. al. (2002); y Alonso (2003) entre otros.

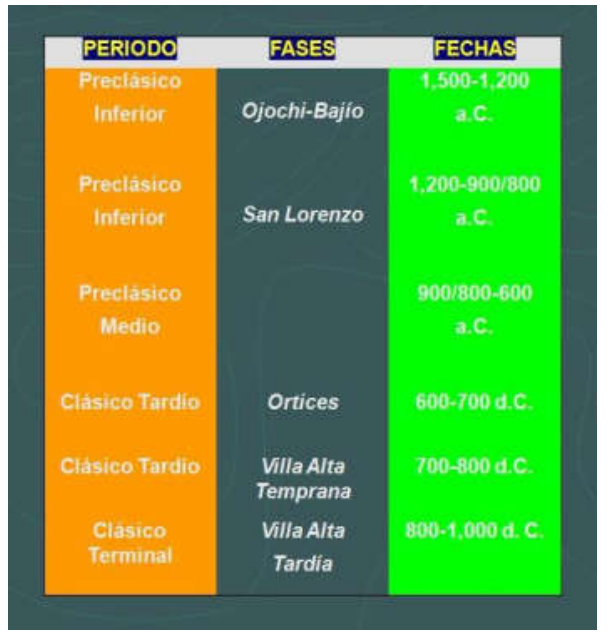

Figura 2: Cronología de la región de Medias Aguas, Veracruz. 
Los asentamientos identificados presentan una variedad de características arquitectónicas y componentes ocupacionales que se pueden fechar desde el periodo Preclásico Inferior: fases Ojochi-Bajío (1,200-900/800 a.C) hasta el periodo Clásico Terminal: Fase Villa Alta Tardía (800-1000 d.C), según la cronología propuesta por Symonds et. al. (2002) para la región vecina de San Lorenzo Tenochtitlán, distante a $30 \mathrm{~km}$ en línea recta hacia el noroeste (Figura 2).

El sitio principal es conocido en la literatura arqueológica por el monumento tallado en basalto denominado "El Mascarón de Medias Aguas" (Figura 3), descubierto en la década de los años cuarenta del Siglo XX por los lugareños; décadas después Medellín Zenil (1960, 1971), reportó por primera vez el sitio al trasladar el monumento al Museo de Antropología de Xalapa en el año 1959; años más tarde, Gómez Rueda (1996) efectuó una recolección de materiales en superficie, así como un croquis del sitio; de tal manera que este sitio y su región no habían sido estudiados sistemáticamente antes de la investigación que se presenta.

Este estudio evidenció un fuerte Patrón de asentamiento sobre la cordillera transversal con dirección este-oeste entre los cerros El Mixe y Medias Aguas, esta banda transversal de tierras elevadas corresponde al límite sur de una intumescencia de origen salino (Masson 2001), permitiendo la subsistencia de asentamientos al margen de las zonas bajas inundables de tipo pantanoso. Este entorno fue el lugar de residencia temporal y permanente de asentamientos humanos que se adaptaron exitosamente a este lugar para lograr una subsistencia 
que les permitió tener relaciones desiguales y posible dependencia con lugares de mayor desarrollo sociopolítico como las regiones vecinas de San Isidro, San Lorenzo y Laguna de los Cerros.

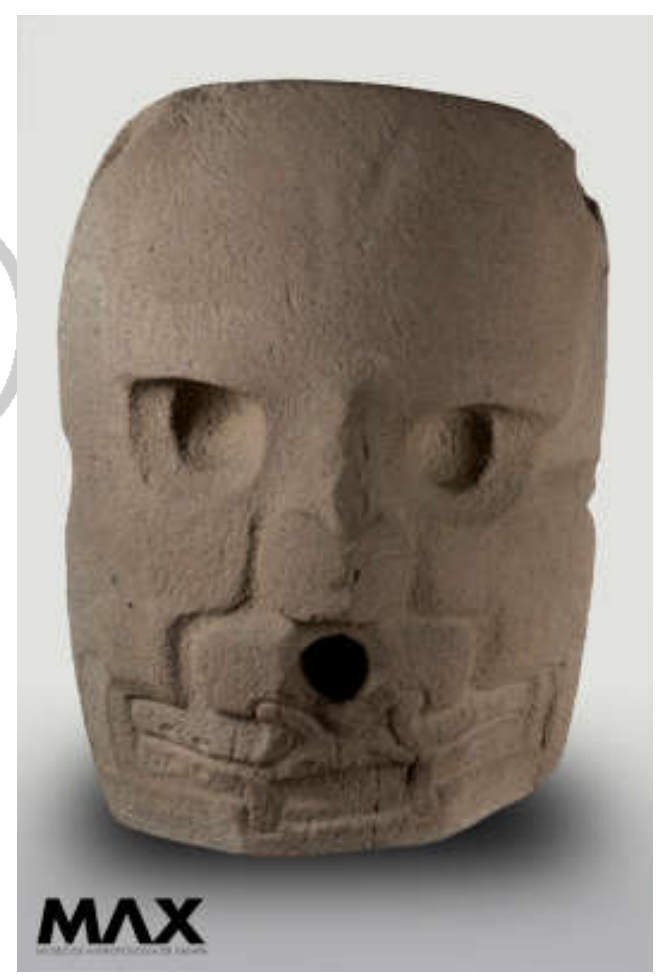

Figura 3: El mascarón de Medias Aguas, Veracruz (cortesía de Hirokazu Kotegawa).

Otros estudios de Patrón de asentamiento en regiones vecinas (Borstein 2001; Symonds et al. 2002), indican que las poblaciones indígenas tuvieron un comportamiento cultural marcado por las relaciones intrínsecas de adaptación a su medio ambiente más cercano. Es notable mencionar, advirtiendo en no caer en el 
determinismo ambiental dogmático, que en la escala espacio-temporal, los cambios (colonización, explosión $\mathrm{y} / \mathrm{o}$ estabilidad) demográficos pueden ser influidos hasta cierto punto por los factores ambientales como se ha señalado para otras regiones mesoamericanas como el valle de Oaxaca (Kowalewski 2003).

La sedimentación producida por el aluvión en zonas inundables, la cobertura vegetal y la erosión producida por agentes naturales y culturales pudieron inhibir el registro de los sitios en el área de estudio; y en consecuencia ofrecer una panorámica distinta del Patrón de asentamiento, seguramente subestimado en términos de densidad poblacional, tendencia similar en el cercano hinterland de San Lorenzo (Symonds et al. 2002; Cyphers 2012).

A grandes rasgos, el poblamiento prehispánico en la región de Medias Aguas se originó con las primeras etapas de ocupación fechadas durante el periodo Preclásico Inferior-fases Ojochi-Bajio (1,500-1,200 a.C.), las cuales estuvieron caracterizadas por un incipiente crecimiento poblacional con la fundación de 50 asentamientos diferenciados en cuatro tipos de sitios (Caserios pequeños, Caseríos medianos, una Aldea pequeña y una Aldea grande) con un notable énfasis por asentarse sobre tierras elevadas, en contraste con la preponderancia por los sitios contemporáneos del tipo Islote asentados en zonas de inundación en la vecina región de San Lorenzo Tenochtitlán (Symonds et al. 2002: 53; 


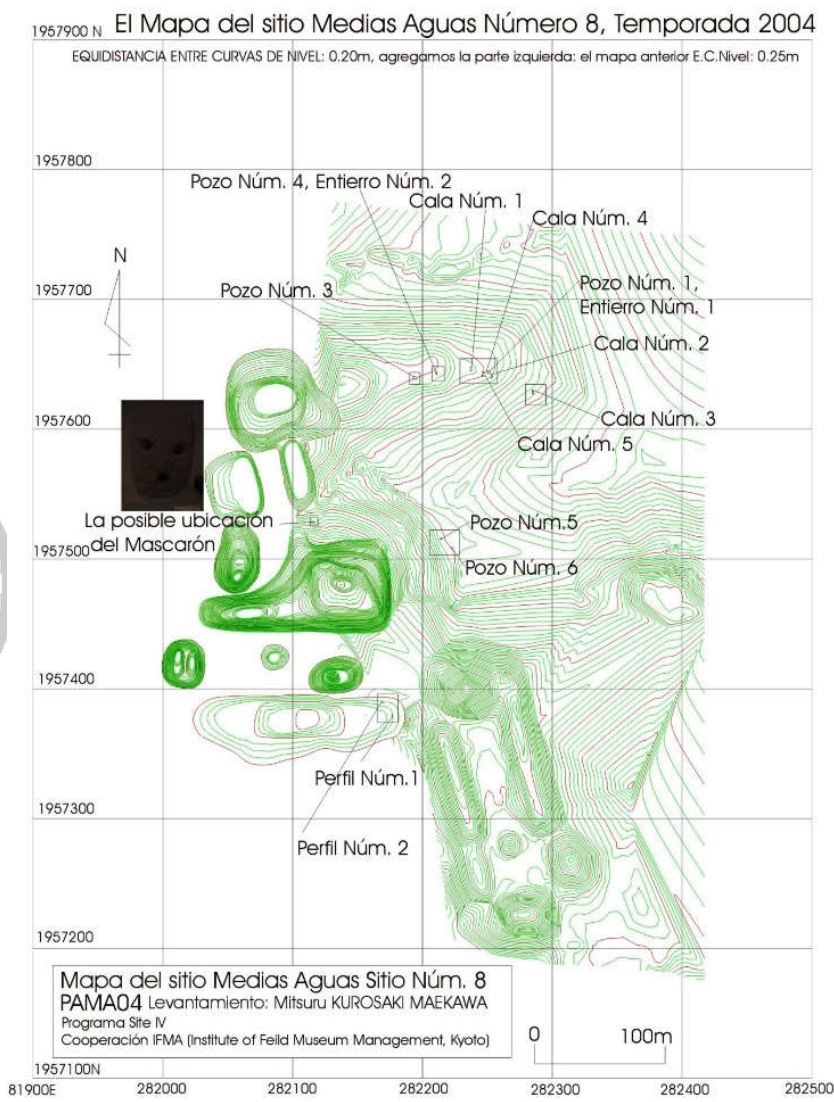

Figura 4: Mapa del sitio Medias Aguas, Veracruz (cortesía de Mitsuru Kurosaki).

Cyphers et al. 2013), y que también han sido considerados como "islas cercanas a las concentraciones de recursos de subsistencia y las rutas de transporte fueron factores significativos en el incremento poblacional y el desarrollo de la complejidad sociopolítica" posterior durante la fase San Lorenzo (Cyphers et al. 2013). 
La región de Medias Aguas presentó una colonización compuesta en su mayoría por sitios de carácter estacional en 40 Caseríos pequeños, así como una menor proporción de sitios permanentes en seis caseríos medianos, una Aldea pequeña (sitio 68-La Isla) y tres Aldeas grandes (sitios 8-Medias Aguas, 35-Los Turrent y 63-El Cházaro), estas últimas ubicadas estratégicamente en puntos altos defensivos, próximos a manantiales de agua y equidistantes entre sí (entre 6 a 7 $\mathrm{km}$ aproximadamente) evidenciando una posible competencia entre jefes locales, quienes pudieron controlar el acceso a los recursos cercanos o a las labores de extracción de los mismos, organizando los medios de

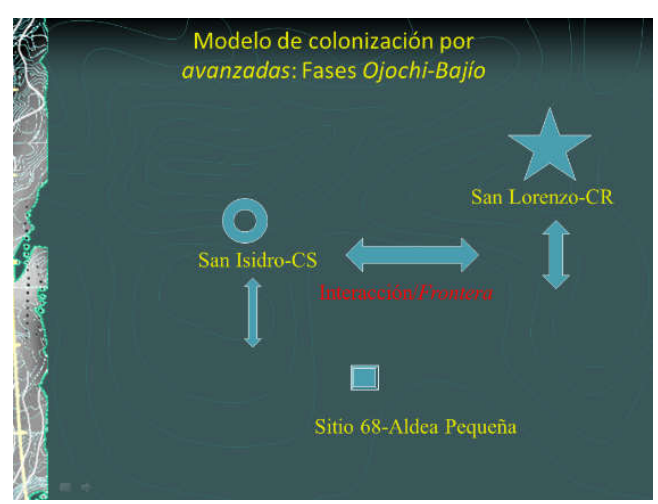

Figura 5: Modelo de colonización.

producción y actuando como mediadores en intercambios para consolidar su poder con residencia sobre nodos estratégicos. 
Las Aldeas grandes y pequeña parecen significar puestos de avanzadas de San Lorenzo o San Isidro habitados con gente común con posibles interacciones de frontera del tipo horizontal concebida como "áreas vírgenes abiertas al crecimiento y colonización" (Feinman \& Nicholas 1990). Estas avanzadas son evidenciadas a partir de indicadores arqueológicos como la presencia de los tipos cerámicos (Acamaya roja, Caimán pulido y Rojo chipo) y características similares como tamaño, densidad de materiales y modificaciones del terreno; a las de los sitios del hinterland de San Lorenzo en el mismo momento (Symonds et al. 2002).

Por otra parte, la mayoría de los sitios asentados sobre los lomeríos que conducen como ruta terrestre al cerro El Mixe, hablan de una marcada tendencia a la ocupación sobre tierras elevadas próximas a manantiales de agua y libres de inundación, tendencia contraria con el hinterland de San Lorenzo durante la misma fase (Symonds et al. 2002: 53; Cyphers 2012).

Para la fase siguiente de San Lorenzo (1,200-900/800 a.C.), la población creció de forma extraordinaria fundándose 54 sitios nuevos para un total de 119 asentamientos en su gran mayoría de carácter estacional (101 caseríos pequeños) y sólo ocho sitios de carácter permanente emplazados sobre la rampa acumulativa de sedimentos manteniéndose la tendencia al asentamiento sobre zonas elevadas, (trece caserios medianos y continúa ocupada la Aldea pequeña (sitio 68-La Isla) que se encuentra muy próxima al estero Monte Obscuro, corriente fluvial más 
importante en la región. Lo que le confiere una posición especial cercana a esta posible ruta de comunicación y transporte; por otro lado también se mantienen ocupadas las mismas aldeas grandes: sitios 8-Medias Aguas, 35-Los Turrent y 63-El Cházaro, sumándose el sitio 99-Campo Nuevo, enclavadas estratégicamente sobre los lomeríos y distanciadas regularmente.

Los tiestos cerámicos recuperados en los sitios se aprecian sólo algunos tipos propios de San Lorenzo: Garza alisado, Caolín, Tigrillo y Zaura; así como decoraciones "elites" del tipo Calzadas excavado, Limón inciso y Cocción diferencial controlada y no controlada propias del Tipo Tigrillo. Esto podría evidenciar posibles interacciones de periferia bajo el desarrollo de dominio de un “centro nuclear" (Feinman \& Nicholas 1990), pero vinculados a través de canales específicos de comunicación de carácter vertical a un centro secundario cercano como San Isidro o de forma directa con un centro rector más distante como San Lorenzo.

Entre las similitudes entre las regiones de Medias Aguas y San Lorenzo podemos argumentar un fuerte desarrollo poblacional con una ligera variedad de cuatro tipos de sitios (caseríos pequeños y medianos, una Aldea pequeña y Aldeas grandes) sin llegar a una compleja jerarquía socio-política al estilo de San Lorenzo caracterizado por ocho tipos de sitios desde pequeños islotes hasta un Centro regional o Capital (Symonds et al. 2002; Cyphers 2012, Cyphers et al. 2013). 


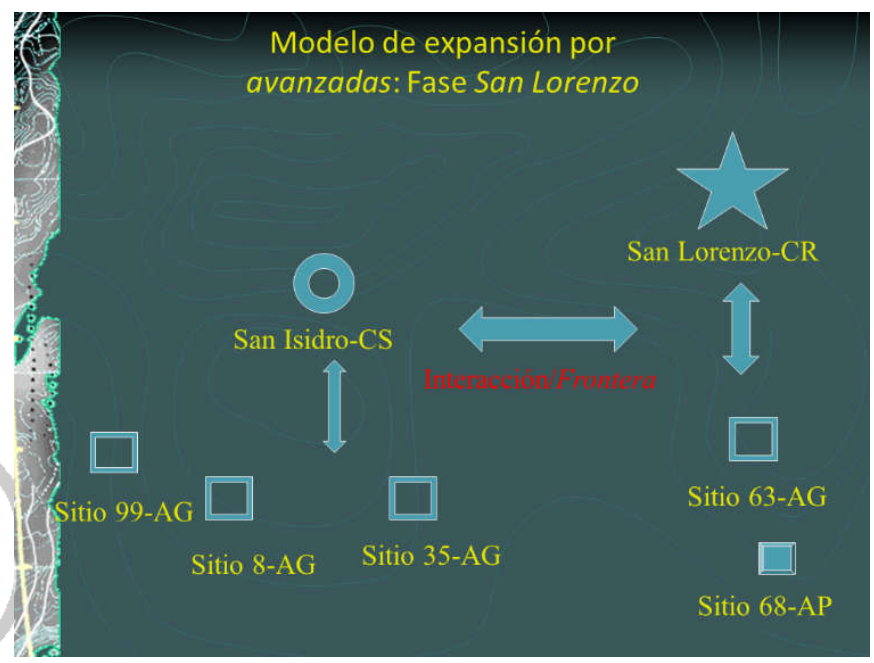

Figura 6: Modelo de expansión.

En cuanto a las diferencias, el Patrón de asentamiento mayoritariamente se presentó sobre los lomeríos en Medias Aguas, a diferencia de San Lorenzo que se dio de forma compartida tanto en zonas inundables como en una extensa cordillera de tierras elevadas, pero de eminentemente carácter estacional (Symonds et al. 2002). Por otra parte en la región de Medias Aguas, no hay evidencia de monumentos de roca como posibles marcadores de expansión sociopolítica presentes en centros secundarios como San Isidro (Cyphers 2004; 2008), aunque se ha propuesto recientemente la "reutilización del Mascarón de Medias Aguas" de un posible pequeño trono a un monumento del periodo Clásico (Ladrón de Guevara 2010). 
Es importante notar que la fase San Lorenzo presentó la mayor densidad poblacional durante toda la ocupación prehispánica en la región de Medias Aguas propiciado posiblemente por migraciones provenientes de una zona nuclear o formación estatal como San Lorenzo. Esto refleja la presencia de avanzadas como indicadores de un sistema económico hegemónico que se extiende más allá del control directo político de una zona nuclear como San Lorenzo o San Isidro.

Bajo la premisa que la expansión económica y el incremento de la urbanización de "estados tempranos" requieren la incorporación de recursos no locales (Algaze 1993) como las rocas volcánicas y metamórficas para la escultura monumental y arte lapidario, así como la obsidiana como materia prima para la manufactura de instrumentos y artefactos. Sin embargo para la región de Medias Aguas, existe poca o nula evidencia de las relaciones informales de carácter económico en la redistribución de bienes y/o productos (pigmentos rojos para la decoración de vasijas y repellado de estructuras arquitectónicas, rocas areniscas, dolomíticas, y maderas tropicales como materiales constructivos, y la sal para el consumo humano) como una vía eficiente de intercambio entre zonas geográficas distantes por medio de la creación de avanzadas para la obtención de recursos de zonas periféricas y su distribución en nodos nucleares de manufactura de bienes de prestigio como San Lorenzo (Coe \& Diehl 1980; Cyphers 1997, 2012; Di Castro y Cyphers 2006; Symonds et al. 2002). 
El objetivo de la fundación de avanzadas de sociedades estatales pudo ser la obtención y redistribución de recursos administrados por una élite foránea residente en San Lorenzo a través de relaciones asimétricas. La incorporación de nuevas áreas a la expansión de centros nucleares o estados tempranos prístinos mediante la fundación de avanzadas lejanas en áreas periféricas representaron una repentina estrategia complementaria a una alta organización de los centros nucleares como San Isidro y San Lorenzo, explotando diferentes sociedades estructuradas y sus alrededores como los cientos de sitios de Medias Aguas.

Las avanzadas pueden ser entendidas como un reflejo de un Sistema Mundial temprano basado en procesos sistemáticos de intercambio asimétrico e interdependencia transcultural como lo han argumentado (e.g. Algaze 1993; Blanton \& Feinman; Chase-Dunn 1979; Kohl 1987).

En consecuencia, San Lorenzo como estado emergente, pudo establecer las avanzadas en la periferia como puntos de mediación entre grupos desiguales, localizadas en puntos clave de comunicación y transporte, así como en la confluencia de redes de intercambio intra-regional e inter-regional, implantadas en la cumbre de jerarquías pre-existentes desde las fases Ojochi-Bajío en las Aldeas grandes (sitios 8-Medias Aguas, 35-Los Turrent, 63-El Cházaro y sitio 99-Campo Nuevo) y durante esta fase San Lorenzo, como instrumentos de expansión para procurar la explotación de recursos orquestado para el desarrollo de políticas regionales de estrategia expansionista. 
Para Algaze (1993), la expansión imperial y la formación de estados tempranos prístinos van de la mano. La posible incorporación de nuevas áreas hacia un núcleo en expansión y la fundación de avanzadas lejanas en áreas periféricas, representa una estrategia complementaria explosiva por parte de una política nuclear altamente organizada, dígase San Lorenzo, explotando diferencialmente sociedades estructuradas en sus alrededores, es decir Medias Aguas.

De ese modo, las avanzadas representan un sistema hegemónico económico que se extendió más allá de sus áreas bajo el control político directo (Algaze 1993); en este sentido, las avanzadas pueden ser entendidas como el reflejo de un temprano Sistema Mundial, basado de alguna manera en un proceso sistemático de intercambio asimétrico y de interdependencia transcultural (Algaze 1993; Piot 1992).

Si la tendencia ocupacional se dio preponderantemente sobre las tierras elevadas a salvo de las inundaciones de las corrientes fluviales, ésta debió emplear a la agricultura y la recolección como base de su subsistencia; en contraste con el exitoso empleo de la pesca y explotación de recursos lacustres en la región inundable de la llanura norte de San Lorenzo (Symonds et al. 2002: 121; Cyphers et al. 2013).

Por su parte (Borstein 2001: 289), menciona que en la región de Laguna de los Cerros se dio un Patrón de asentamiento tanto en zonas de tierras bajas, así como 
en tierras altas; esta tendencia es una propuesta compartida en parte con la región de Medias Aguas, donde la adaptación de la población del periodo Preclásico se dio en gran medida sobre las tierras elevadas a salvo de las inundaciones.

Durante esta fase de ocupación, se puede reconocer la importancia socio-política de dos sitios con notoria supremacía regional: San Isidro fungiendo como un Centro secundario cercano y San Lorenzo convertido en un Centro regional más lejano. Ambos sitios basaron su supremacía mediante el control de ideas, bienes y/o productos, etc. en la región de Medias Aguas y quizá hacia territorios más distantes como el Istmo de Tehuantepec y los Tuxtlas (Cobean 1996; Gómez Rueda 1996; Stark \& Arnold 1997; Symonds et al. 2002; Santley 2007).

En consecuencia, la región de Medias Aguas pudo integrarse al hinterland de San Lorenzo como parte de un sistema hegemónico o Sistema Mundial a través de una o varias rutas de comunicación que fluían en el norte del Istmo de Tehuantepec hacia regiones vecinas y distantes como los Tuxtlas, la costa central del Golfo, la costa del Pacífico, el Centro de México y Centroamérica.

Por otra parte, durante el periodo Preclásico Medio (900/800-600 a.C.), la región de Medias sufrió al igual que otras regiones cercanas como San Lorenzo y Laguna de los Cerros (Borstein 2001; Kilion \& Urcid 2001; Symonds et al. 2002), una marcada restricción poblacional: 107 sitios son abandonados de la fase previa y se fundan sólo 18 asentamientos con un reducido orden en dos tipos de sitios: 16 
Caseríos pequeños de carácter estacional y sólo dos Caseríos medianos de carácter permanente (sitio 2-Cerro de Medias Aguas y el sitio 49); esta escasa población se asentó otra vez predominantemente en los lomeríos de manera estacional y por lo tanto, la región revela una tendencia de abandono similar al hinterland de San Lorenzo (Symonds et al. 2002).

Los indicadores arqueológicos que revelan la temporalidad de los sitios son la presencia en casi todos los sitios de dos tipos cerámicos (Tigrillo y Zaura) con formas propias del Preclásico Medio como vasijas con labio caído y evertido, silueta compuesta y decoración zonal con incisiones en sitios como San Lorenzo, La Venta e Isla Alor, y Arroyo Pesquero (Di Castro y Cyphers 2006; Symonds et al 2002; González 2001; Wendt \& Lunagómez 2011).

Al parecer se reconoce una posible interacción de frontera de carácter horizontal a través de los dos únicos sitios permanentes: los caseríos medianos ((sitio 2-Cerro de Medias Aguas y el sitio 49). Se abandonaron la mayoría de los sitios ocupados desde la fase previa de San Lorenzo, y en consecuencia no existe ninguna evidencia de avanzadas durante esta fase, sino más bien un extenso abandono intra-regional e inter-regional, efecto demográfico que transformó a la región de Medias Aguas en una frontera casi vacía. 


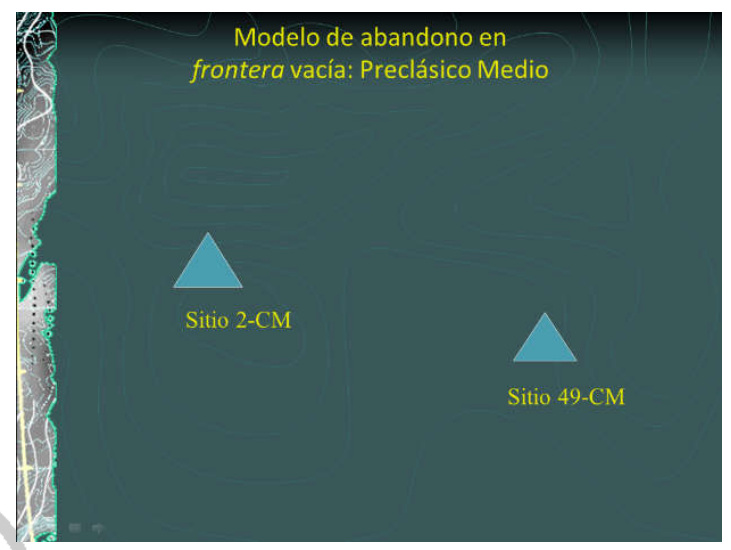

Figura 7: Modelo de abandono.

En contraste, Urcid y Killion (2004: 11) mencionan que para fines del periodo Formativo Temprano y principios del Formativo Medio en la región de Hueyapan se presentó un notable incremento de asentamientos.

Tal parece que durante el periodo Preclásico Medio, la región de Medias Aguas, así como las regiones vecinas de San Lorenzo Tenochtitlán y Laguna de los Cerros compartieron la nula presencia/influencia de centros poblacionales de primer orden cercanos, debido posiblemente a la hegemonía socio-política de un centro lejano como La Venta y sitios de su hinterland como Arroyo Pesquero, San Andrés, Isla Alor, etc. (Rust \& Sharer 1988; González Lauck 1994, 2001; Raab et al. 2001; Pohl et al. 2002; Von Nagy 1997; Wendt \& Lunagómez 2011).

El abandono de las regiones de San Lorenzo Tenochtitlán y Laguna de los Cerros entre los periodos Preclásico Medio y Clásico Tardío aún no ha podido explicarse del todo (Symonds et al. 2002: 94-96; Borstein 2001: 211). Aunque se pueden 
contemplar posibles alteraciones medio ambientales, como la actividad volcánica en la región de los Tuxtlas (Cyphers 1997; Malmstrom 2002; Symonds et al. 2002; Santley 2007) y transgresiones de la costa marítima del Golfo de México (Hammond 1988), como factores naturales que incidieron directa o indirectamente en el Patrón de asentamiento regional. En general, para el territorio del sur de Veracruz, la transición ocupacional entre los periodos Preclásico y Clásico, caracterizada por un "fuerte despoblamiento" para algunos autores ha sido probablemente exagerado (Pool 2006: 200).

La región de Medias Aguas, no fue ajena a este proceso de "aparente abandono", no se tiene registro de ningún tipo de sitio con materiales fechados posteriores al periodo Preclásico Medio hasta finales del periodo Clásico.

En suma, diversas hipótesis acerca de este extenso fenómeno de abandono se agrupan de la siguiente manera: primero, la afectación directa o indirecta de los factores medio ambientales sobre la población; segundo, una marcada modificación en las rutas de intercambio hacia y con otras áreas mesoamericanas (Stark \& Arnold 1997; Symonds et al. 2002) y la nucleación hacia otras regiones o centros poblacionales como los Tuxtlas, la Mixtequilla y el Centro de Veracruz (Stark 2001; Daneels 2002; Santley 2007); tercero, la imposibilidad de reconocer tradiciones y complejos cerámicos utilizados como marcadores cronológicos (Pool 1995); y por último, una propuesta de explicación multifactorial como drásticos $\mathrm{y} / \mathrm{o}$ paulatinos cambios medio ambientales aunados a fuertes 
mecanismos culturales como migraciones masivas, guerras, epidemias, cambios de rutas de intercambio, emergencia de nuevos centros socio-políticos, etc.

Después de un prolongado hiato de ocupación de casi un milenio, la región de Medias Aguas al igual que otras regiones del sur de Veracruz (Gómez Rueda 1996; Borstein 2001; Symonds et al. 2002), presenciaron una nueva actividad poblacional como posible consecuencia de migraciones procedentes desde otras regiones de Mesoamérica (Scholes y Roys 1996; Lunagómez 2011; Symonds et al. 2002). Esta nueva ocupación para la región de Medias Aguas, se fecha en la fase Ortices (600-700 d.C.) durante el periodo Clásico Tardío. La población de esta fase se distribuyó en 16 asentamientos divididos nuevamente en sólo dos tipos de sitios: 12 Caseríos pequeños y sólo cuatro Caseríos medianos (sitios 4, 7, 19 y 41).

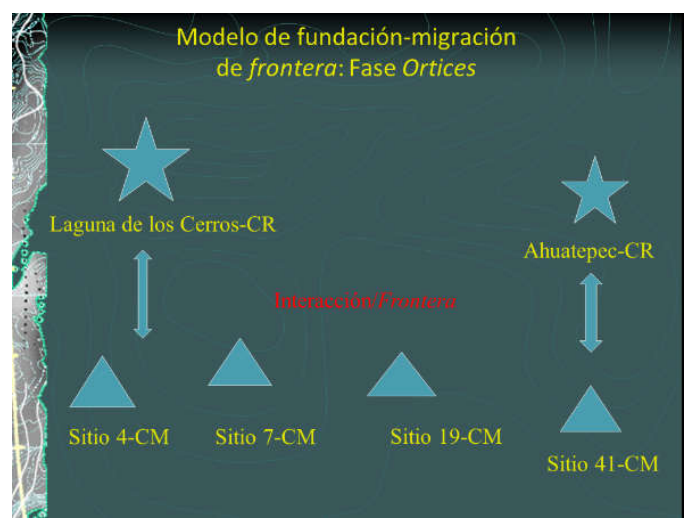

Figura 8: Modelo de fundación-migración. 
El Patrón de asentamiento revela nuevamente un carácter estacional, tendencia quizá heredada desde el periodo Preclásico; en este momento se hace sentir la presencia/influencia de nuevos centros rectores: Ahuatepec ubicado muy lejos al noreste de Medias Aguas en la isla de Tacamichapa en la vertiente occidental del río Coatzacoalcos; y por otra parte, probablemente el sitio más extenso de todo el periodo Clásico en el Sur de Veracruz: Laguna de los Cerros, que aunque desde el periodo Preclásico ya era un centro secundario a San Lorenzo (Bové 1978; Borstein 2001), en este momento alcanzó la supremacía regional evidenciada en su extensa y compleja arquitectura monumental.

Tiempo después, quizá un siglo, durante la fase Villa Alta Temprana (700-800 d.C.), la región volvió progresivamente a aumentar y complejizar su población, reflejada en la ocupación de 54 asentamientos divididos en cuatro tipos de sitios: 42 caseríos pequeños, nueve caseríos medianos, dos aldeas grandes (sitios 63-El Cházaro y 99-Campo Nuevo) y un Centro secundario (sitio 8-Medias Aguas). Los sitios 63-El Cházaro y 99-Campo Nuevo se transformaron en aldeas grandes con arquitectura monumental arreglada en plazas; y el sitio 8-Medias Aguas, se convirtió en un Centro secundario, quizá subordinado a Laguna de los Cerros dada sus cercanía, por sus características únicas en la región como: extensa área de ocupación en 40 ha, la arquitectura monumental arreglada en tres grandes plazas y la presencia de una escultura en roca (el mascarón de Medias Aguas). 
A partir de esta fase de ocupación en el sur de Veracruz, inició la construcción de la arquitectura monumental arreglada en plazas rodeadas de montículos alargados rematados en ambos lados por montículos cónicos, también llamado "Arreglo Cuatripartito Villa Alta-VAQA" (Borstein 2001: 37), "Conjunto Plaza" (Domínguez 2001: 104), "Plaza Monumental” (Daneels 2002: 173) y/o "patrones arquitectónicos" (Lunagómez 2011).

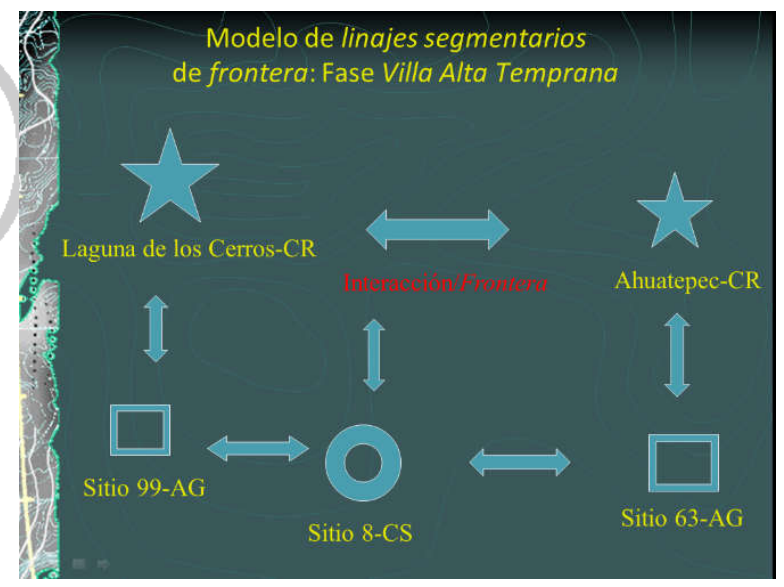

Figura 9: Modelo de linajes segmentarios de frontera.

Para la última parte del periodo Clásico Tardío, durante la fase Villa Alta Tardía (800-1,000 d.C.), tanto Ahuatepec localizado al noreste de la región de San Lorenzo Tenochtitlán, así como Laguna de los Cerros (con 123 montículos y 300 ha de área) se convirtieron respectivamente en un Centro secundario y regional con arquitectura monumental a gran escala y extenso tamaño (Borstein 2001: 218; Symonds et al. 2002), evidenciando una fuerte influencia socio-política sobre otros sitios como San Lorenzo, Tenochtitlán, San Isidro, La Oaxaqueña, Las 
Limas, Medias Aguas, etc. (Coe \& Diehl 1980; Cobean 1996; Gómez 1996; Borstein 2001; Lunagómez 2011; Symonds et al. 2002).

Tanto para Borstein (2001: 211) la fase Villa Alta representó "el pico de la densidad ocupacional prehispánica en el sur de Veracruz", así como para Symonds y otros (2002: 105) la fase Villa Alta Tardía también representó el pináculo de la ocupación prehispánica en la región de San Lorenzo Tenochtitlán.

En contraste en la región de Medias Aguas, la fase Villa Alta Tardía (800-1,000 d.C.) del periodo Clásico Terminal fue la ocupación de segunda importancia después de la población de la fase San Lorenzo.

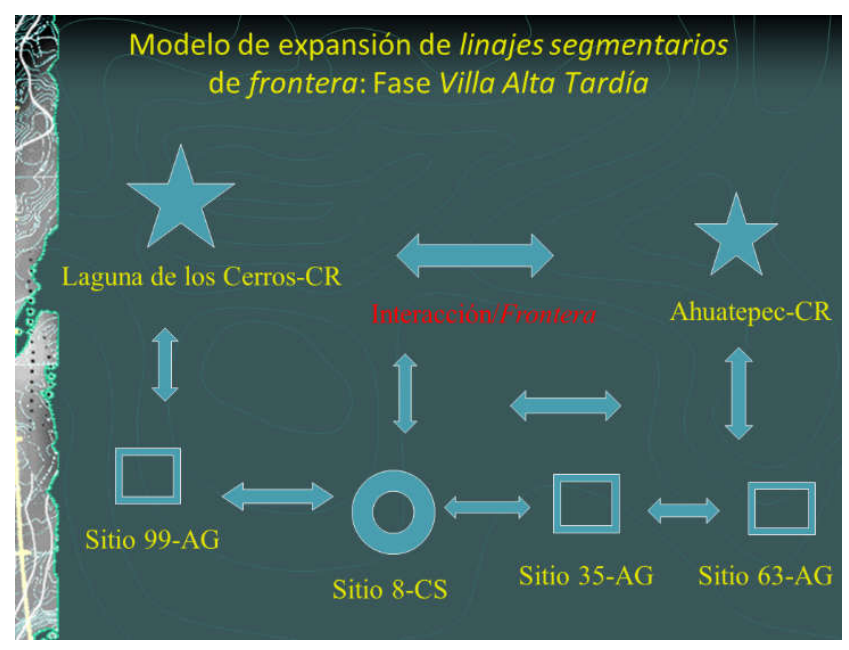

Figura 10: Modelo de expansión de linajes segmentarios de frontera. 
La población nuevamente se incrementó en forma extraordinaria en 106 asentamientos con cuatro tipos de sitios: 90 caserios pequeños, 12 caseríos medianos, tres aldeas grandes (sitios 35-Los Turrent, 63-El Cházaro y 99-Campo Nuevo) y un Centro secundario (sitio 8-Medias Aguas). Sin embargo, el Patrón de asentamiento revela otra vez un carácter estacional, tendencia heredada desde el periodo Preclásico y mantenida durante el Clásico, posiblemente como una exitosa estrategia de subsistencia y/o de interacción con otros centros rectores o regiones vecinas: Laguna de los Cerros, Ahuatepec, etc. Los sitios 63-El Cházaro y 99-Campo Nuevo se mantuvieron como aldeas grandes con arquitectura monumental arreglada en plazas integrando posiblemente una población dispersa a su alrededor como las decenas de caseríos medianos, y sólo el sitio 35-Los Turrent se transformó en una Aldea grande.

El sitio 8-Medias Aguas se consolidó como el único Centro secundario de la región por sus atributos señalados (gran tamaño, arquitectura monumental y escultura en roca); tal parece que la región fue "influenciada" por sitios de mayor complejidad socio-política fuera del área de estudio como Laguna de los Cerros al noroeste y Ahuatepec al noreste (Borstein 2001; Lunagómez 2011; Symonds et al. 2002).

Durante la parte final del periodo Clásico en Medias Aguas, ¿Cuáles pudieron ser las estrategias de subsistencia emprendidas y los mecanismos de integración socio-política? 
Una alternativa viable pudo ser el modo de producción de los llamados "linajes segmentarios" (Fox 1988; Daneels 2002; Lunagómez 2011) basado en el control de las tierras y la producción de alimentos tanto en tierras altas como en zonas inundables por una élite regida por el parentesco mediante mecanismos de integración socio-política (voluntarios y/o coercitivos) evidenciada en la complejidad de la arquitectura monumental (arreglo formal, planeamiento urbano, orientación cardinal, conjuntos arquitectónicos: plazas, canchas de pelota, etc.) característica del periodo Clásico en el sur de Veracruz.

A manera de epílogo, desde las primeras ocupaciones humanas del periodo Preclásico Inferior (fases Ojochi-Bajio y San Lorenzo) hasta las últimas ocupaciones (fases Villa Alta Temprana y Tardía), la región de Medias Aguas pudo considerarse interrelacionada con la hegemonía de centros de alta jerarquización socio-política como San Lorenzo y San Isidro durante el periodo Preclásico; y de Laguna de los cerros y Ahuatepec durante los periodos Clásico Tardío y Terminal. Este argumento se respalda por el hecho de que ningún sitio en la región alcanzó las características físicas, ni un orden mayor a los asentamientos reportados para las regiones mencionadas como tamaño de sitio, arquitectura monumental y escultura en roca (Gómez Rueda 1996; Borstein 2001; Lunagómez 2011; Symonds et al. 2002). 
En el entorno fisiográfico de la región de Medias Aguas, los factores más importantes para la ubicación de los sitios se dieron a partir del acceso a las tierras fértiles para la agricultura, la disponibilidad de manantiales de agua potable, la defensa en contra de enemigos locales o foráneos y el resguardo de las inundaciones periódicas que ofrecían las tierras elevadas: este entorno seguramente influyó en el Patrón de asentamiento, condicionando las aglomeraciones de sitios en terrenos más aptos para la subsistencia y protección. En las tierras elevadas se puso en práctica sistemas de cultivo intensivos (quizá milpas) evidenciados por el terraceo y otros tipos de modificación de lomeríos naturales complementada por otras actividades como la pesca, caza y recolección. Los habitantes de los sitios más importantes seleccionaron los puntos más elevados y favorables del entorno terrestre con fines de comunicación, defensa, transporte e intercambio desde las primeras hasta las últimas fases de ocupación prehispánica.

La presencia en las "mejores tierras o tierras elevadas" de los sitios de gran tamaño y arquitectura monumental arreglada en plazas propios los periodos Clásico Tardío y Terminal como "controladores" de las rutas de paso, parece indicar la incorporación de jerarquías socio-políticas en la región de otros sitios más importantes en zonas cercanas como San Isidro, San Lorenzo Tenochtitlán, Laguna de los Cerros y Ahuatepec. 
La adaptación y explotación de su medio ambiente inmediato (riberas de ríos, esteros y lagunas por una parte y tierras elevadas por otra), fue el sustento de la complejidad socio-política desde los Olmeca hasta los pobladores de los periodos Clásico Tardío y Terminal.

En la región vecina de San Lorenzo Tenochtitlán (Symonds et al. 2002: 60), la subsistencia se basó en el ambiente ribereño y lacustre que requería de estrechos mecanismos de integración socio-política en comparación con las tierras elevadas en la región de Medias Aguas. En donde estas tierras elevadas funcionaron como un verdadero "corredor o puente" en el tránsito de gente de una región a otra, señalado por la gran distribución y concentración de sitios de diferentes tipos y rangos a través de los distintos momentos de ocupación antigua.

Según Borstein (2001: 227) durante el periodo Clásico Terminal la extensión poblacional cubrió y explotó una mayor variedad de ecozonas (mosaicos de tierras altas y planicies inundables) en la región de Laguna de los Cerros, lo que contrasta con el fuerte aprovechamiento de los ambientes ribereños durante el periodo Preclásico. En oposición en la región de Medias Aguas, los asentamientos cubrieron en forma mayoritaria las tierras elevadas desde el periodo Preclásico hasta el Clásico Terminal, lo cual sugiere que la población se adaptó y se condicionó por las características particulares de su entorno más cercano. 
Llama la atención que la fase San Lorenzo (1,200-900/800 a.C.), esté mejor representada en el registro arqueológico, que la ocupación más reciente de la fase Villa Alta Tardía (800-1,000 d.C.), lo cual sugiere la importancia de este momento durante toda la ocupación prehispánica en la región de estudio. Esta importancia estuvo sustentada en la hegemonía socio-política que ejerció San Lorenzo sobre su hinterland, dentro del cual Medias Aguas mantuvo un papel clave por su posición estratégica funcionando como una encrucijada de caminos hacia los cuatro puntos cardinales: al norte la costa del Golfo de México, al sur la costa del Pacífico, al oriente las planicies inundables del sur de Veracruz y Tabasco, y al oeste la ruta hacia el Altiplano Central.

Es de tomarse en cuenta que dentro del ámbito macro-regional en la parte septentrional del Istmo de Tehuantepec, la región de Medias Aguas cumplió y cumple una función estratégica en la migración humana desde el pasado hasta el día de hoy, por ser una ruta obligada en el paso entre los lomeríos y los humedales que le dan su nombre: "en medio de las aguas". 


\section{REFERENCIAS BIBLIOGRÁFICAS}

ALGAZE, Guillermo

1993 "Expansionary Dynamics of Some Early Pristine States" American Anthropologist, New Series, Vol. 95, No. 2, American Anthropological Association, pp. 304-333.

ALONSO, Alejandra, 2003 "Estudio Arqueológico en el Cerro de La Encantada, Veracruz". Tesis de Maestría en Antropología especialidad Arqueología, Facultad de Filosofía y Letras-División de Estudios de Posgrado-Instituto de Investigaciones Antropológicas-UNAM, C. U. México D. F.

BLANTON, Richard \& Gary Feinman, 1984 "The Mesoamerican World System" American Anthropologist, New Series, Vol. 86, No. 3, American Anthropological Association, pp. 673-682.

BORSTEIN, Joshua A, 2001 Tripping over colosal heads: Settlement patterns and population development in the upland olmec heartland. Ph. Dr. diss., The Pennsylvannia State University, State College.

BOVÉ, Frederick J, 1978 Laguna de los Cerros: An Olmec Central Place. Journal of New World Archaeology, Volume II, Number 3, Institute of Archaeology, University of California, Los Angeles.

CHASE-DUNN, Christopher K, 1979 "Comparative Research on World-System Charateristics" International Studies Quarterly, Vol. 23, No. 4, International Studies Association, pp. 601-623.

COBEAN, Robert H, 1996 "La Oaxaqueña, Veracruz: un centro olmeca menor en su contexto regional". Arqueología Mesoamericana. Homenaje a William T. Sanders. Mastache, Alba Guadalupe; Jeffrey R. Parsons; Robert S. Santley y Mari Carmen Serra Puche. (coords.), Tomo 2, p. 37-61, INAH-Arqueología Mexicana, México. 
COE, Michael D. \& Richard A. Diehl, 1980 In the Land of the Olmec: Vol. 1: The Archaeology of San Lorenzo Tenochtitlán. Vol. 2: The People of the River. University of Texas Press, Austin \& London.

CYPHERS, Ann, 1997 Población, Medio Ambiente y Subsistencia en San Lorenzo Tenochtitlán. Instituto de Investigaciones Antropológicas-UNAM, México.

2004 Escultura Olmeca de San Lorenzo Tenochtitlán. Instituto de Investigaciones Antropológicas-UNAM, México.

2008 "Los tronos y la configuración del poder olmeca", Ideología política y sociedad en el periodo Formativo, Ensayos en homenaje al doctor David C. Grove, A. Cyphers y K. Hirth, eds., p. 311-341, Instituto de Investigaciones Antropológicas, Universidad Nacional Autónoma de México, México.

2012 Las bellas teorías y los terribles hechos, Controversias sobre los olmecas del Preclásico inferior. Instituto de Investigaciones Antropológicas, Universidad Nacional Autónoma de México.

CYPHERS, Ann, Judith Zurita Noguera y Marci Lane Rodríguez, 2013 Retos y riesgos en la vida Olmeca. Instituto de Investigaciones Antropológicas, UNAM, México.

DANEELS Verriest, Annick J. E, 2002, “El Patrón de Asentamiento del Periodo Clásico en la Cuenca Baja del río Cotaxtla. Centro de Veracruz: Un estudio de caso de sociedades complejas en tierras bajas tropicales". Tesis de Doctorado en Antropología especialidad Arqueología, Facultad de Filosofía y Letras-División de Estudios de Posgrado-Instituto de Investigaciones Antropológicas-UNAM, C. U. México D. F.

DI CASTRO, Anna y Ann Cyphers, 2006, "Iconografía de la cerámica de San Lorenzo" Anales del Instituto de Investigaciones Estéticas, otoño, año/vol. XXVIII, número 089, Universidad Nacional Autónoma de México, pp. 29-58. 
DOMÍNGUEZ Covarrubias, Elba, 2001 "La arquitectura monumental del periodo Clásico en el sur de Veracruz: un enfoque regional". Tesis de licenciatura en Antropología con especialidad en Arqueología. Departamento de Antropología, Universidad de Las Américas, Puebla, Cholula.

FEINMAN, Gary M. \& Linda M. Nicholas, 1990, "At the margins of the Monte Alban state: Settlement pattterns un the Ejutla Valley, Oaxaca, Mexico" Latin American Antiquity, Vol. 1, No. 30, Society American Archaeology, pp. 216-246.

FOX, John W, 1988 "Hierarchization in Maya segmentary states". State and Society: The emergence and development of social hierarchy and political centralization. Gledhill, John, Barbara Bender \& Mogens Trolle Larsen. Academic Division of Unwin Hyman Ltd, London.

GÓMEZ Rueda, Hernando, 1996 Las Limas, Veracruz, y otros asentamientos prehispánicos de la región Olmeca. Colección Científica, $\mathrm{n}^{\circ}$ 324, Serie Arqueología, INAH, México.

GONZÁLEZ Lauck, Rebecca, 2001 'Investigaciones arqueológicas en 'isla' Alor: un sitio en el área de sostenimiento de La Venta, Tabasco". Arqueología, 2a . Época, No. 26, pp. 3-14. Coordinación Nacional de Arqueología. Instituto Nacional de Antropología e Historia, México.

HAMMOND, Norman, 1988, "Cultura hermana: Reappraising the Olmec". The Olmec and the Development of Formative Mesoamerican Civilization. Sharer, Robert J. \& David C. Grove (eds). A school of American Research Book, Cambridge University Press, Cambridge \& New York.

KILLION, Thomas W. \& Javier Urcid, 2001, "The Olmec Legacy: Cultural Continuity and Change in Mexico's Southern Gulf Coast Lowlands". Journal of Field Archaeology, Vol. 28, p. 3-25.

KOHL, Philip L, 1987, "The Use and Abuse of Word System Theory: The Case of the Pristine West Asian State" Advances in Archaeological Method and Theory , Vol. 11, Springer, pp. 1-35. 
KOWALESKI, Stephen A, 2003, "Scale and the Explanation of Demographic Change: 3,500 years in the Valley of Oaxaca" American Anthropologist, Vol. 105, No. 2, American Anthropological Association, pp. 313-325

KRUGER, Robert P, 1996 An Archaeological Survey in the Region of the Olmec, Veracruz, Mexico. Ph. Dr. diss., Deparment of Anthropology, University of Pittsburgh, Pittsburgh, PA.

LADRÓN de Guevara González, Sara, 2010, "Reutilización de monumentos olmecas en tiempos del clásico" Ancient Mesoamerica, Vol. 21, No. 1, Cambridge University Press, pp. 63-68.

LUNAGÓMEZ Reyes, Roberto, 2011, Los patrones arquitectónicos prehispánicos del sur de Veracruz: Epoca Clásica. Cuerpo Académico “Arqueología del paisaje y cosmovisión", Universidad Veracruzana, Programa del Mejoramiento del Profesorado-PROMEP, Xalapa.

MALMSTROM, Vincent H, 2002, "La Chontalpa: ¿tierra de nadie o cuna de la civilización maya?".Arqueología, No. 27, Revista de la Coordinación Nacional de Arqueología del INAH, Segunda Epoca, enero-junio, INAH, p. 5-14, México.

MASSON, Pierre, 2001, Influencia de la tectónica salina sobre la geomorfología en la cuenca salina del istmo veracruzano y sus consecuencias sobre el ambiente habitacional y cultural de los olmecas y de culturas ulteriores. Ponencia presentada en el II Foro de Arqueología, Facultad de Antropología de la Universidad Veracruzana, Xalapa.

MEDELLÍN Zenil, Alfonso, 1960 "Monolitos inéditos olmecas". La Palabra y El Hombre. No. 16: p. 75-97. Universidad Veracruzana, Xalapa.

1971 Monolitos olmecas y otros en el Museo de Antropología de la Universidad Veracruzana. Unión Academique Internacionale. INAH, México.

MÜNCH Galindo, Guido, 1983 Etnología del Istmo Veracruzano. Instituto de Investigaciones Antropológicas-UNAM, México. 
PIOT, D. Charles, 1992, "Wealth Production, Ritual Consumption, and center/periphery relations in a West African regional system" American Ethnologist, Vol. 19, No. 1, American Anthropological Association, pp. 34-52.

POOL, Christopher A, 2006 "Current Research on the Gulf Coast of Mexico". J Archaeol Res, 14, p. 189-241, Springer Science + Bussiness Media, Inc.

SANTLEY, Robert S, 2007 The Prehistory of the Tuxtlas. University of New Mexico Press, Albuquerque.

SCHOLES, France V. y Ralph L. Roys. 1996, Los chontales de Acalan-Tixchel. Centro de Investigaciones y Estudios Superiores en Antropología Social y Centro de Estudios Mayas, México.

STARK, Barbara L, 2001, Classic Period Mixtequilla, Veracruz, México: Diachronic Inferences from Residential Investigations. Institute for Mesoamerican Studies, Monograph 12, University at Albany, Albany, New York.

STARK, Barbara \& Philip J. Arnold III (eds.), 1997, "Introduction to the Archaeology of the Gulf Lowlands". Olmec to Aztec: Settlement Patterns in the Ancient Gulf Lowlands. University of Arizona Press, p. 3-32, Tucson.

1997 Olmec to Aztec: Settlement Patterns in the Ancient Gulf Lowlands. University of Arizona Press, Tucson.

SYMONDS, Stacey, Ann Cyphers y Roberto Lunagómez, 2002, Asentamiento Prehispánico en San Lorenzo Tenochtitlán. Serie San Lorenzo, Coordinadora: Ann Cyphers, Volumen 2, UNAM, Instituto de Investigaciones Antropológicas y Dirección General de Asuntos del Personal Académico, México.

WENDT, Carl \& Roberto Lunagómez, 2011, "Investigating the Arroyo Pesquero Olmec". Mexicon, Zeitschrift für Mesoamerikaforschung-Revista sobre Estudios Mesoamericanos, Vol. XXXIII, Nr. 3, p. 73-79, Juni 2011, Deuchtland-Alemania. 